making them higher risk of shoulder injury. Preventive strategies are frequently used to decrease injuries. However, prior to implementing a preventive program could be effective identify the athletes' beliefs and opinions about injury prevention to increase the engagement.

Objective To describe the perceptions on preventive strategies among overhead recreational athletes.

Design Cross sectional study.

Setting An online semi-structured survey on a group of recreational overhead athletes.

Participants Fifty-one recreational overhead athletes (male $=34$; female $=17$; $31.16 \pm 10.82$ years; mean sport experience $=14.3$ years) took part in the online survey. Of those participants, 13 were from basketball (25.5\%), 11 from handball $(21.6 \%)$, 16 from tennis (31.4\%), and 11 from volleyball (21.6\%).

Assessment of Risk Factors An online semi-structured survey was applied, with the following categories: (1) history of injuries, (2) self-reported preventive strategies. Descriptive statistics and qualitative research methods were used to perform a thematic analysis.

Main Outcome Measurements Self-reported injury prevalence; beliefs on preventive strategies; Categories resulting from the thematic analysis, with (1) self-reported preventive strategies, (2) professional support to preventive strategies.

Results Overall, 42 athletes (82.4\%) had experienced a sportinjury in the past. For 48 athletes $(94.1 \%)$ is possible to prevent injuries and the self-reported preventive strategies were: 'muscle strengthening' ( $\mathrm{n}=19)$, 'muscle stretching' $(\mathrm{n}=10)$, 'neuromuscular preventive exercises' $(n=7)$, 'warm-up exercises' $(n=6)$, 'adjustment of movements' $(n=5)$, 'higher cardiovascular resistance' $(\mathrm{n}=5)$, 'use of protective equipment' $(n=3)$, 'nutrition management' $(n=2)$, and 'rest' $(n=2)$. Besides, forty athletes self-reported the physiotherapist like the professional to support preventive strategies $(n=40)$, followed by physical educator $(n=19)$, personal trainer $(n=16)$, and coach $(n=4)$.

Conclusions In conclusion, the self-report preventive strategies from overhead athletes are in partial agreement with scientific evidence. This study identified athlete beliefs that could be incorporate in future preventive programs.

\section{THERE WAS A HIGH INCIDENCE OF MATCH INJURIES AT THE 2019 INTERNATIONAL NETBALL WORLD CUP COMPETITION, MOSTLY IN THE LOWER LIMB AND FOLLOWING CONTACT WITH OTHER PLAYERS}

\footnotetext{
${ }^{1,2}$ Christa Janse van Rensburg, ${ }^{3,4}$ Grace Bryant, ${ }^{1}$ Audrey Jansen van Rensburg, ${ }^{5,6}$ Sharon Kearney, ${ }^{2,7}$ Praimanand Singh, ${ }^{8}$ Arnold Devos, ${ }^{1}$ Martin Schwellnus, ${ }^{9}$ Tanita Cronje. 'Sport, Exercise Medicine and Lifestyle Institute (SEMLI) and Section Sports Medicine, Faculty of Health Sciences, University of Pretoria, Pretoria, South Africa, Pretoria, South Africa; ${ }^{2}$ Committee member of the Medical Committee of INF, Global, South Africa; ${ }^{3}$ Sports Medicine at Sydney University, University of Sydney, Australia, Sydney, Australia; ${ }^{4}$ Chair of the Medical Commission of INF, Global, Australia; ${ }^{5}$ NetballSmart Programme Manager Netball New Zealand, New Zealand, New Zealand; ${ }^{6}$ Vice President Sports Medicine New Zealand, New Zealand, New Zealand; 'Lecturer in Sports Medicine and Consultant in Emergency Medicine at the University of the West Indies, West Indies, -; ${ }^{8}$ Principal Consultant, Background Signal P/L, Australia, Sydney, Australia; ${ }^{9}$ Department of Statistics, University of Pretoria, South Africa, Pretoria, South Africa
}

\subsection{6/bjsports-2021-IOC.353}

Background Netball is a fast action game and there is growing interest in the sport. The Netball World Cup (NWC) is an international netball world championship, contested every four years, with 16 teams qualifying by ranking and play-offs. Research on netball injuries is however limited.

Objective To determine the incidence and severity of injuries during the 2019 NWC.

Design Prospective study, cross sectional analysis.

Setting Netball World Cup 2019 in Liverpool, England.

Patients (or Participants) 192 elite female netball players from all 16 contesting teams at the NWC (average squad size 12 players).

Interventions (or Assessment of Risk Factors) Medical staff of each team recorded all injuries (all- and match injuries; 840 total exposure hours) and training/match days lost during the 10-day tournament.

Main Outcome Measurements Incidence rate (IR) (per 1000 player-hours; 95\% CI) and severity (\% time-loss $\geq 1$ day) of all injuries and match injuries.

Results 48 independent injuries were recorded (46 during match-play, 2 during training) in 192 players (25\%). The IR of match injuries was 54.8 (38.9-70.6). The IR in the lower limb $(28.6 ; 17.1-40.0)$ was significantly higher $(p=0.016)$ vs. the head $(9.5 ; 2.9-16.1 ; \mathrm{p}=0.016)$ and upper $\operatorname{limb}(8.3 ; 2.2-$ 14.5; $\mathrm{p}=0.002)$. In the lower limb, the IR of ankle injuries was significantly higher than knee injuries $(p=0.033)$. Most injuries (71\%) occurred in contact situations with another athlete with $34 \%$ occurring during quarter 3 of the game, followed by quarter 1 and 2 (26\% each). 28\% of all injuries were time-loss injuries. The $\%$ injuries, by player position, were: centre (25\%), goal keeper $(21 \%)$ and goal defence (19\%).

Conclusions There was a high incidence of injuries in elite netball players, with 1 in 4 players sustaining an injury. There is an urgent need to introduce targeted injury prevention strategies in netball internationally to reduce the risk of injuries during the NWC.

\section{MORE THAN 80\% OF ILLNESSES AT THE 2019 INTERNATIONAL NETBALL WORLD CUP COMPETITION ARE ONLY REPORTED ON MATCH DAY: IS THERE NOT A NEED TO EDUCATE PLAYERS?}

\begin{abstract}
${ }^{1}$ Audrey Jansen van Rensburg, ${ }^{1,2}$ Christa Janse van Rensburg, ${ }^{3,4}$ Grace Bryant, ${ }^{5,6}$ Sharon Kearney, ${ }^{2,7}$ Praimanand Singh, ${ }^{8}$ Arnold Devos, ${ }^{1}$ Martin Schwellnus, ${ }^{9}$ Tanita Cronje. 'Sport, Exercise Medicine and Lifestyle Institute (SEMLI) and Section Sports Medicine, Faculty of Health Sciences, University of Pretoria, Pretoria, South Africa, Pretoria, South Africa; ${ }^{2}$ Committee member of the Medical Committee of INF, Global, South Africa; ${ }^{3}$ Sports Medicine at Sydney University, University of Sydney, Australia, Sydney, Australia; ${ }^{4}$ Chair of the Medical Commission of INF, Global, Australia; ${ }^{5}$ NetballSmart Programme Manager Netball New Zealand, New Zealand, New Zealand; ${ }^{6}$ Vice President Sports Medicine New Zealand, New Zealand, New Zealand; 'Lecturer in Sports Medicine and Consultant in Emergency Medicine at the University of the West Indies, West Indies, -; ${ }^{8}$ Principal Consultant, Background Signal P/L, Australia, Sydney, Australia; ${ }^{9}$ Department of Statistics, University of Pretoria, South Africa, Pretoria, South Africa
\end{abstract}

\subsection{6/bjsports-2021-IOC.354}

Background The international Netball World Cup (NWC) is contested every four years and 16 teams qualify to participate, based on their world ranking. The risk, nature and severity of illness at the NWC is not known.

Objective To determine the incidence, severity and reporting of illness by players during the NWC in 2019.

Design Prospective study, cross sectional analysis.

Setting Netball World Cup 2019, Liverpool, England. 